The Open Respiratory Medicine
Journal
JrossMark
Content list available at: www.benthamopen.com/TORMJ/
DOI: $10.2174 / 1874306401610010020$

\title{
Relationship Between Lung Cancer and Mycobacterium Avium Complex Isolated Using Bronchoscopy
}

\author{
Atsuhisa Tamura, ${ }^{1,2,}$, Akira Hebisawa ${ }^{2}$, Kei Kusaka ${ }^{1}$, Takashi Hirose ${ }^{1}$, Junko Suzuki ${ }^{1}$, Akira \\ Yamane $^{1}$, Hideaki Nagai ${ }^{1}$, Takeshi Fukami ${ }^{3}$, Ken Ohta ${ }^{1}$ and Fumiaki Takahashi ${ }^{4}$ \\ ${ }^{I}$ Center for Pulmonary Diseases and Respiratory Disease Division, National Hospital Organization Tokyo National \\ Hospital, Kiyose, Tokyo 204-8585, Japan \\ ${ }^{2}$ Clinical Research Center and Pathology Division, National Hospital Organization Tokyo National Hospital, Kiyose, \\ Tokyo 204-8585, Japan \\ ${ }^{3}$ Center for Pulmonary Diseases and Chest Surgery Division, National Hospital Organization Tokyo National Hospital, \\ Kiyose, Tokyo 204-8585, Japan \\ ${ }^{4}$ Clinical Research, Innovation and Education Center, Tohoku University Hospital, Sendai, Miyagi 980-8574, Japan
}

\section{Abstract:}

\section{Introduction:}

The incidence of Mycobacterium avium complex (MAC)-positive respiratory specimen cultures and MAC lung disease (MACLD) is increasing worldwide. This retrospective study aimed to assess the association between MAC culture-positive bronchoscopy specimens and lung cancer.

\section{Materials and Methods:}

The medical records of 1382 untreated lung cancer patients between 2003 and 2011 were collected using our hospital database. Of them, records for 1258 that had undergone bronchoscopy together with sampling for mycobacterial culture were reviewed. Patient characteristics were compared between those with MAC-positive/other nontuberculous mycobacteria (NTM)-negative bronchial washings and those with MAC-negative/other NTM-negative bronchial washings. Patients with MAC-positive lung cancer were cross-sectionally divided into MACLD and non-MACLD groups, and their features were assessed. Follow-up data for patients with lung cancer but without MACLD were reviewed for subsequent development of MACLD.

Results:

Of the 1258 patients with lung cancer, 25 (2.0\%) had MAC-positive/other NTM-negative bronchial washings. The proportion of women $(52 \%$ vs $30 \% ; P=0.0274)$ and patient age (72 years $v s 69$ years; $P=0.0380$ ) were significantly higher in the MACpositive/other NTM-negative lung cancer group $(\mathrm{n}=25)$ than in the MAC-negative/other NTM-negative lung cancer group $(\mathrm{n}=$ 1223). There were 10 patients with lung cancer and MACLD and 15 without MACLD; significant differences in patient characteristics were not found between the two groups, and none of the 15 patients without MACLD subsequently developed MACLD.

\section{Conclusion:}

MAC culture-positive bronchial washing is positively associated with lung cancer. Female sex and advanced age, but not lung cancer characteristics, were found to be associated with MAC infection in patients with lung cancer.

Keywords: Advanced age, Bronchoscopy, Culture, Female, Lung cancer, Mycobacterium avium complex, Mycobacterium avium complex lung disease.

\footnotetext{
* Address correspondence to this author at the Center for Pulmonary Diseases and Respiratory Disease, National Hospital Organization Tokyo National Hospital, 3-1-1 Takeoka, Kiyose, Tokyo 204-8585, Japan; Tel: +81-424-91-2111; Fax: +81-424-94-2168; E-mail: tamura-in@tokyo-hosp.jp
} 


\section{INTRODUCTION}

Nontuberculous mycobacteria (NTM)-associated lung disease (NTMLD) is a major cause of morbidity and mortality, with a steadily increasing worldwide incidence $[1,2]$. The mycobacteria species isolated in NTMLD vary geographically; however, Mycobacterium avium complex (MAC) is the most frequently isolated species in many countries [2 - 4]. In Japan, MAC lung disease (MACLD) accounts for approximately $80 \%$ of all NTMLDs [4, 5]. Various factors are associated with NTMLD, including environmental factors and host factors, such as thoracic skeletal abnormalities, rheumatoid arthritis, previous pulmonary tuberculosis, bronchiectasis, chronic obstructive pulmonary disease, and lung cancer $[2,6]$. Lung cancer is a comorbidity in $4-7 \%$ of patients with respiratory NTM (primarily MAC) isolates [7, 8]. However, MACLD in patients with lung cancer has rarely been investigated [9]. Recently, Lande et al.[10]. reported that $25 \%(62 / 249)$ of patients with lung cancer have respiratory cultures positive for MAC. It is important to gain insight into the relationship between MAC-positive cultures and lung cancer. However, patients with pulmonary diseases, including lung cancer, often do not have sputum-associated symptoms; thus, sputum examination for acid-fast bacilli is often not conducted for lung cancer cases. Indeed, in a recent report, sputum culture for mycobacteria was only performed in $15 \%$ (249/1632) of patients with lung cancer [10]. Therefore, current estimates of the sputum culture MAC-positivity rate in lung cancer is based on incomplete or inaccurate data.

In Japan, fiberoptic bronchoscopy is usually performed to obtain a definitive diagnosis of MACLD as well as pulmonary tuberculosis [11 - 13] in sputum-negative patients. Furthermore, because Japan is a middle-ranked country for pulmonary tuberculosis prevalence [14], bronchial washings collected during bronchoscopy are routinely cultured for mycobacteria to diagnose pulmonary diseases, including lung cancer. Although cross-sectional frequency using MAC-positive cultures in bronchial washings is not intended to reflect the overall frequency of MAC positivity in lung cancer, comorbid lung cancer is simultaneously diagnosed in the majority of patients with MAC-positive cultures [9, 10]. In most lung cancer patients, bronchoscopy is performed at diagnosis; thus, simultaneous diagnosis of the two diseases may be obtained by bronchoscopy. Therefore, in considering the MAC-positive rate in lung cancer, assessment of MAC-positive bronchoscopy cultures is important.

The aim of the present retrospective study was to assess the relationship between MAC-positive bronchoscopy samples and lung cancer.

\section{METHODS}

\section{Patients and Clinical Setting}

We retrospectively reviewed a total of 1,382 consecutive cases of lung cancer admitted to the National Hospital Organization, Tokyo National Hospital between January 2003 and December 2011. Of these patients, 1,258 met the inclusion criteria: 1) initial bronchoscopy was performed to obtain a diagnosis of lung cancer with sampling for mycobacterial culture; 2) chest computed tomography (CT) to increase diagnostic accuracy was undertaken before bronchoscopy; and 3) bacterial, radiographic, pathological, and other clinical data were available from the medical records. Thus, mycobacterial data from the initial bronchoscopy with sampling for mycobacterial culture were analyzed. The case flow diagram is shown in Fig. (1).

There were 106 patients who did not undergo bronchoscopy because of a poor performance status and a definite diagnosis using other samples such as malignant pleural effusions. CT images from before bronchoscopy and other various data were obtained from our hospital databases (lung cancer database, bacteriological database, and other medical records).

\section{Sample Collection and Diagnosis}

Fiberoptic bronchoscopy was carried out by experienced physicians via the transoral or transnasal route under local anesthesia and sedation according to the standard procedures: 1) complete inspection of the tracheobronchial trees, including subsegmental bronchi; 2) sampling with bronchial brushings of the bronchial segments at which the target lesion was located on CT; 3) sampling of bronchial and/or lung biopsy from the same segments; and 4) collection of bronchial washings at the orifices of the same segment and related lobe.

Fresh samples of bronchial washings were submitted to the bacteriology and pathology divisions of our facility. For bacteriology, part of each sample was prepared as smears on glass slides and was examined for acid-fast bacilli. The remaining sample was subjected to rapid genetic diagnosis of mycobacteria using a polymerase chain reaction (PCR; 
Roche, Basel, Switzerland) assay until May 2009 and using a transcription-reverse transcription concerted reaction (TRC; Tosoh, Tokyo, Japan) assay starting in June 2009. For samples that were positive for Mycobacterium tuberculosis on PCR and/or TRC, no further genetic tests were performed. For samples that were negative for $M$. tuberculosis on PCR and/or TRC, PCR for M. avium and M. intracellulare or TRC for MAC was performed. All samples with or without positive PCR and/or TRC results were cultured for mycobacteria using mycobacteria growth indicator tubes (BACTEC MGIT 960: Becton, Dickinson and Company, Franklin Lakes, NJ, USA). Finally, mycobacterial species were identified using DNA-DNA hybridization (Kyokutoseiyaku, Tokyo, Japan). DNA-DNA hybridization of NTM-positive samples was entrusted to the physicians.

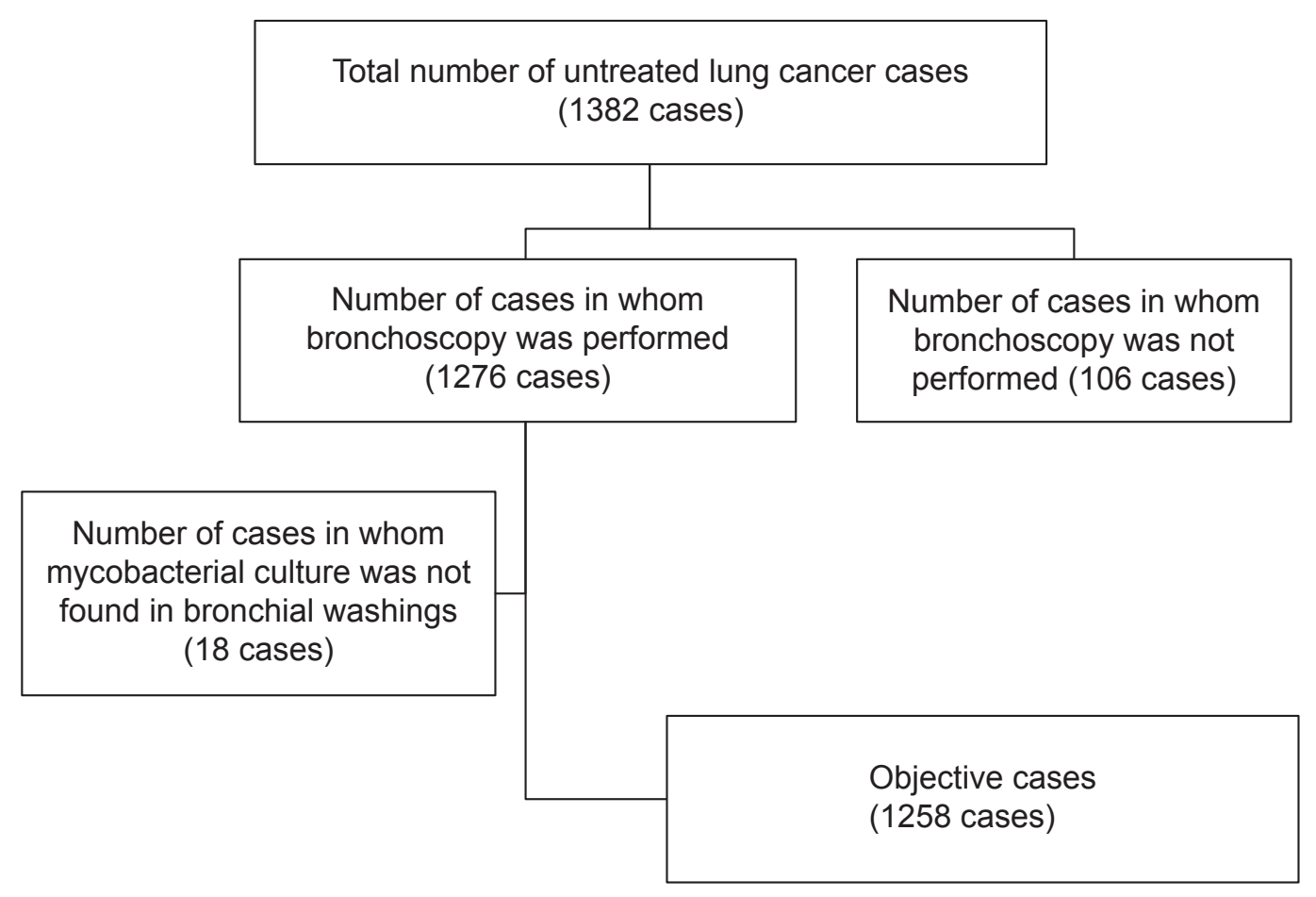

Fig. (1). Flow diagram of patients with lung cancer receiving bronchoscopy between 2003 and 2011.

At our hospital, bronchoscope contamination and associated infection are prevented by sterilization with an endoscope reprocessor (OER-2; Olympus, Tokyo, Japan) containing 0.3\% ethaneperoxoic acid (Acecide; Saraya, Osaka, Japan), which is a very effective mycobactericidal agent for disinfecting bronchoscopes [15].

\section{Data Compilation and Analysis}

The patients were divided into MAC-positive/other NTM-negative group, MAC-negative/other NTM-positive group, and MAC-negative/other NTM-negative group. Based on the NTMLD criteria (two positive sputum cultures or one bronchoscopy or lung biopsy culture with clinical and radiographic features) of the American Thoracic Society and Infectious Diseases Society of America [16], the MAC-positive group and other NTM-positive group were further cross-sectionally divided into MACLD/NTMLD (with characteristic radiographic features) and non-MACLD/ NTMLD (without characteristic radiographic features) groups.

We investigated the lung cancer status at the time of the confirmation of the culture results from bronchial washings in MAC-positive/other NTM-negative patients. The lung cancer status included surgical data after the bronchoscopy. Subsequently, the features of MAC-positive/other NTM-negative lung cancer patients and MAC-negative/other NTMnegative lung cancer patients were compared and analyzed using our hospital database. Next, in the MAC-positive/other NTM-negative lung cancer patients, we compared the lung cancer status between the MACLD lung cancer patients and the non-MACLD lung cancer patients. In addition to the cross sectional analyses, follow-up data for lung cancer patients with MAC-positive non-MACLD were analyzed for subsequent development of MACLD. 


\section{Statistical Analysis}

All statistical analyses were conducted using R Statistical Software version 3.0.2. Intergroup differences were evaluated using univariate analysis by means of the independent samples t-test for continuous variables and the chisquare test or Fisher's exact test for categorical variables, as appropriate. Multivariate analyses were included in the logistic regression analysis and performed using the backward elimination method. Differences were considered statistically significant at $P<0.05$.

Our institutional review board approved the study (December 24, 2013; approval number 130053), and patient informed consent was considered unnecessary. This study was conducted in accordance with the Declaration of Helsinki.

\section{RESULTS}

\section{MAC Positivity in Lung Cancer Patients}

Among the 1,258 patients evaluated, 25 (2.0\%) had MAC-positive/other NTM-negative cultures, $10(0.8 \%)$ had MAC-negative/other NTM-positive cultures, and the remaining 1223 had both negative cultures for MAC and other NTM. Among the 10 MAC-negative/other NTM-positive cases, there were two cases with M. kansasii-positive, two with M. gordonae-positive, one with M. szulgai-positive, and in the remaining five cases, NTM-species were not identified. Only two among $10 \mathrm{M}$. kansasii-positive cases met the NTMLD criteria.

The characteristics of these MAC-positive/other NTM-negative patients are summarized in Table $\mathbf{1 .}$

Table 1. Characteristics of the 25 MAC-positive/other NTM-negative patients with lung cancer.

\begin{tabular}{|l|l|c|}
\hline \multicolumn{2}{|c|}{ Case Number } \\
\hline Sex & & $13 / 12$ \\
\hline Female/Male & & $72(67-78)$ \\
\hline Median age (IQR), years & & $14 / 11$ \\
\hline Smoking & & \\
\hline Yes/No & & $22 / 3$ \\
\hline Lung cancer & & $15 / 1 / 9$ \\
\hline Type & Peripheral/Hilar & $18 / 4 / 3$ \\
\hline Location & UL/ML/LL & $15 / 10$ \\
\hline Histology & AD/SQ/Others & \\
\hline Stage & III-IV/I-II & \\
\hline
\end{tabular}

MAC: Mycobacterium avium complex; IQR: interquartile range; UL: upper lobe; ML: middle lobe; LL: lower lobe; AD: adenocarcinoma; SQ: squamous cell carcinoma.

The predominant clinicopathological features of the 25 lung cancer patients with MAC-positive cultures were female sex $(n=13)$, smoking history $(n=14)$ and advanced age (median age, 72 years; IQR, 67 years-78 years). Lung cancers were predominantly of the peripheral type $(n=22)$, were located in the upper lobe $(n=15)$, were adenocarcinomas based on histology $(n=18)$, and were at clinical stage III-IV $(n=15)$.

Table 2. Univariate analysis of the clinical features of MAC-positive/other NTM-negative lung cancer and MACnegative/other NTM-negative lung cancer.

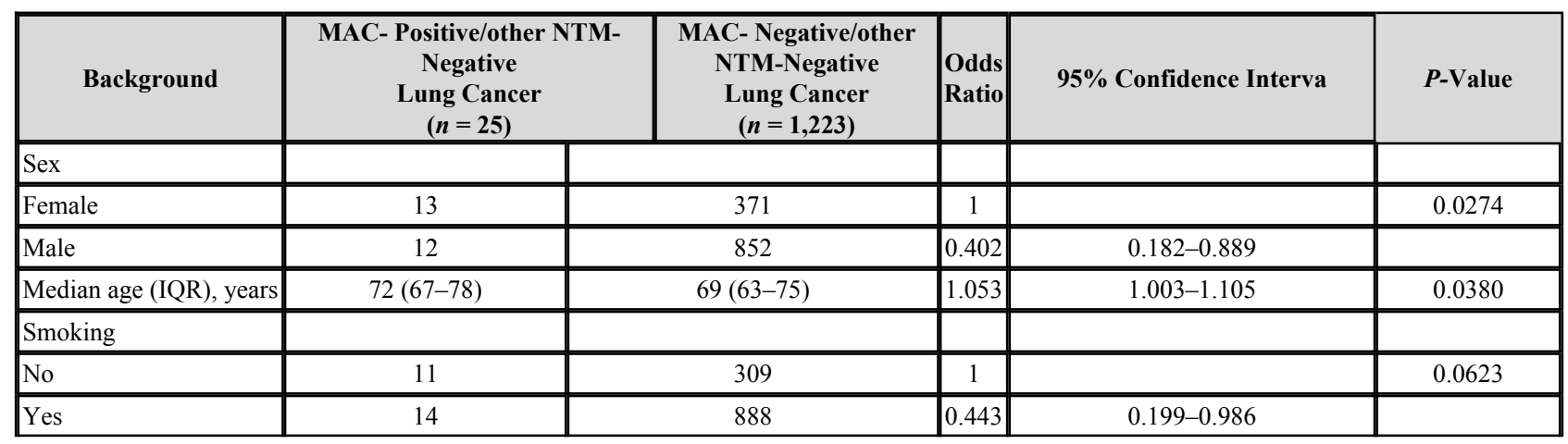




\begin{tabular}{|c|c|c|c|c|c|}
\hline Background & $\begin{array}{c}\text { MAC- Positive/other NTM- } \\
\text { Negative } \\
\text { Lung Cancer } \\
(n=25) \\
\end{array}$ & $\begin{array}{c}\text { MAC- Negative/other } \\
\text { NTM-Negative } \\
\text { Lung Cancer } \\
(n=1,223) \\
\end{array}$ & $\left|\begin{array}{c}\text { Odds } \\
\text { Ratio }\end{array}\right|$ & $\mathbf{9 5 \%}$ Confidence Interva & $P$-Value \\
\hline Unknowna & 0 & 26 & & & \\
\hline \multicolumn{6}{|l|}{ Lung cancer } \\
\hline Hilar & 3 & 307 & 1 & & 0.1637 \\
\hline Peripheral & 22 & 916 & 2.458 & $0.731-8.268$ & \\
\hline Location & 15 & 622 & 1 & & 0.5194 \\
\hline LL & 9 & 482 & 0.774 & $0.336-1.784$ & \\
\hline Others & 1 & 119 & 0.348 & $0.046-2.663$ & \\
\hline Histology & 18 & 731 & 1 & & 0.4651 \\
\hline SQ & 4 & 274 & 0.593 & $0.199-1.767$ & \\
\hline Others & 3 & 218 & 0.559 & $0.163-1.915$ & \\
\hline Stage & 10 & 560 & 1 & & 0.6862 \\
\hline III-IV & 15 & 663 & $|1.267|$ & $0.565-2.842$ & \\
\hline
\end{tabular}

${ }^{\mathrm{a}}$ Data not available. MAC: Mycobacterium avium complex; IQR: interquartile range; UL: upper lobe; LL: lower lobe; AD: adenocarcinoma; SQ: squamous cell carcinoma.

Statistical test was used to calculate the $P$-value.

We compared the 25 patients with lung cancer who were MAC-positive/other NTM negative with the 1223 MACnegative/other NTM-negative patients. In the univariate analysis, the number of women was significantly higher in the MAC-positive lung cancer group than in the MAC-negative lung cancer group $(13 / 25 v s .371 / 1223 ; P=0.0274)$. MACpositive/other NTM-negative patients were also significantly older than MAC-negative/other NTM-negative patients (72 years vs. 69 years; $P=0.0380$ ). In contrast, positive smoking history, lung cancer type, location, histologic type, and clinical stage were not significantly different between the MAC-positive and MAC-negative lung cancer groups (Table 2).

In the multivariate logistic regression analysis, female sex (odds ratio [OR], 0.388; 95\% confidence interval [CI], $0.175-0.860 ; P=0.0198)$ and older age (OR, $1.054 ; 95 \% \mathrm{CI}, 1.005-1.105 ; P=0.0309)$ were associated with MACpositive cultures (Table 3 ).

Table 3. Multivariate analysis (backward elimination variable selection) for MAC-positive/other NTM-negative lung cancer and MAC-negative/other NTM-negative lung cancer.

\begin{tabular}{|c|c|c|c|}
\hline & Odds Ratio & 95\% Confidence Interval & $P$-Value \\
\hline Sex & 0.388 & $0.175-0.860$ & 0.0198 \\
\hline Age & 1.054 & $1.005-1.105$ & 0.0309 \\
\hline
\end{tabular}

Statistical test was used to calculate the $P$-value.

The 25 patients with MAC-positive lung cancer were divided into two groups: those with MACLD $(n=10)$ and those without MACLD $(n=15)$. There were no significant clinicopathological differences between these two groups.

Table 4. Status of the 10 patients with lung cancer and comorbid MACLD.

\begin{tabular}{|c|c|}
\hline & Case Number \\
\hline \multicolumn{2}{|l|}{ Type of MACLD } \\
\hline Nodular/bronchiectatic & 8 \\
\hline Fibrocavitary & 1 \\
\hline Others & 1 \\
\hline \multicolumn{2}{|l|}{ Spread of MACLD } \\
\hline Multiple lobes & 9 \\
\hline Single lobe & 1 \\
\hline \multicolumn{2}{|c|}{ Anatomical relationship between MACLD and lung cancer } \\
\hline Same lobe & 7 \\
\hline Another lobe & 3 \\
\hline \multicolumn{2}{|l|}{ Diagnosis of MACLD and lung cancer } \\
\hline Concurrent & 8 \\
\hline Lung cancer sequential to MACLD & 2 \\
\hline
\end{tabular}


(Table 4) contd....

\begin{tabular}{|c|c|}
\hline 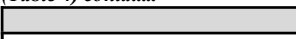 & Case Number \\
\hline Sputum culture for MAC & \\
\hline Positive & 5 \\
\hline Negative & 5 \\
\hline
\end{tabular}

MACLD: Mycobacterium avium complex lung disease.

Details on comorbid MACLD in the 10 lung cancer patients are presented in Table 4.

Eight of the 10 MACLD patients exhibited nodular/bronchiectatic types on CT, and MACLD had spread into multiple lobes in nine cases. As the nodular/bronchiectatic type of MACLD had spread into other lobes from the origin in the middle lobe and lingula, the anatomical relationship between MACLD and lung cancer involved the same lobe in seven patients; however, there were no patients where the two diseases coexisted within the same lesion. Of the 10 patients with MACLD, two had the disease several years before being diagnosed with lung cancer, while for the remaining eight patients lung cancer and MACLD were detected concurrently. Two patients with lung cancer sequential to MACLD and three of the eight concurrent cases also had MAC culture-positive sputum just after bronchoscopy. There were no cases with disseminated MAC diseases.

\section{Follow-up of Non-MACLD Patients with Lung Cancer}

We evaluated the medical data of the 15 patients with lung cancer but not MACLD to determine whether MAC positivity in bronchial washings was a MACLD precursor. All 15 patients with lung cancer received their initial and subsequent lung cancer treatments at our hospital. The median follow-up time was 50 months (range, 37 months-60 months) for 6 patients with stage I-II lung cancer who underwent lobectomy and 12 months (range, 3 months-59 months) for 9 patients with stage III-IV lung cancer who underwent chemotherapy and/or radiotherapy. Among the 6 lobectomy cases, there was only one case with comorbid, non-caseous, epithelioid granulomas surrounding the lung cancer with no acid-fast bacilli in the resected materials; however, the remaining 5 cases had no characteristic MACLD pathologic findings. During the follow-up period, sputum cultures were examined for mycobacteria at least once in all 15 patients. All of the patients had MAC-negative cultures and no radiographic findings compatible with MACLD; accordingly, they did not undergo a second bronchoscopy. Thus, none of the patients had comorbid MACLD.

\section{DISCUSSION}

This retrospective study has revealed for the first time a 2.0\% rate of MAC-positive bronchial specimen cultures in patients with lung cancer with comorbid lung cancer and MACLD observed in $0.8 \%$ of patients with untreated lung cancer who had received bronchoscopy. These results are comparable with our previous report [9].

MAC-positive patients with lung cancer in the present study were more likely to be women, older, and smokers. Further, MAC-positive lung cancers were predominantly peripheral adenocarcinomas, clinical stage III-IV, and located in an upper lobe. However, only female sex and old age were significantly associated with MAC-positive lung cancer when compared with MAC-negative lung cancer patients. Female sex and advanced age, well-known risk factors for MACLD [3, 7, 8, 17], were also associated with MAC infection in patients with lung cancer rather than with lung cancer status.

The clinicopathological features of MAC-positive patients in the present study were generally consistent with those reported by Lande et al. [10], with the exception of the predominance of adenocarcinoma. In our previous study of patients with comorbid lung cancer and NTMLD from 1997 to 2002, smoking history, male sex, squamous cell carcinoma, and fibrocavitary disease on radiographs were predominant clinicopathological features [9]. The predominance of adenocarcinoma in MAC-positive lung cancer patients in our current study might be related to the recent increase in MACLD in Japan. Indeed, the incidence of nodular/bronchiectatic disease in non-smoking females has markedly increased as compared with the previous predominance of fibrocavitary disease in male smokers [8]. A recent Japanese study on comorbid lung cancer in patients with MACLD patients also showed a predominance of females, adenocarcinoma, and the nodular/bronchiectatic type of MACLD [18].

Considering the prevalence of lung cancer and MAC-positive cultures, Daley and Iseman [19] have speculated as to whether or not chronic inflammation caused by MAC infection leads to lung cancer, or if lung cancer allows the establishment of MAC infection. For the latter, MAC is believed to adhere to the non-ciliated epithelial cells in bronchioles at the onset of infection [20], with subsequent granuloma formation causing bronchial destruction and MACLD [21]. West and Dollery [22] have previously described variations in the ventilation-perfusion ratio between the upper and lower lungs and, by means of an analysis of the lung epithelial lining fluid using a bronchoscopy 
microsampling probe, revealed an increase in biomarkers such as carcinoembryonic antigen and cytokeratin fragment 19 concentrations in the fluid around lung cancer foci [23]. We can speculate that lung cancer allows the establishment of an exogenous MAC infection in the bronchioles around the lung cancer foci as a result of some unclear factors associated with the presence of lung cancer itself and/or other pre-existing lung diseases. Our finding that none of the 15 patients with lung cancer and non-MACLD progressed to MACLD may indicate an exogenous MAC infection under conditions suitable for transient infection.

The present study on lung cancer patients does not address lung cancer development associated with MAC infection, but many epidemiological studies have suggested that pulmonary tuberculosis increases the risk of lung cancer [24]. In a pathological study reported in 1939, Friedrich [25] first described a lung cancer that developed from a tuberculous scar, and the presence of scar carcinoma caused by tuberculosis has been historically recognized [26]. The development of lung cancer in NTMLD patients has not been reported in epidemiological studies. However, previous Japanese studies have reported that NTMLD/MACLD and lung cancer were likely to exist in the same lobe [9, 18] and often coexist in the same lesion [27]. The present study also demonstrated that for most of the cases with comorbid MACLD and lung cancer, the two diseases existed in the same lobe. In another study, analysis of the epithelial lining fluid revealed that the inflammatory reaction caused by MAC might spread to an unaffected area other than the original MAC-infected area [28]. Furthermore, attention has been paid to the Mendelian susceptibility to mycobacterial diseases, such as interferon gamma receptor deficiencies and impaired interleukin-2 production, although no data were presented here regarding this [29].

MACLD is a disease with a very long clinical course, and we must keep in mind that chronic inflammation caused by long-term MAC infection may exert some influence on cancer development.

\section{Study Limitations}

The present study had some limitations that are inherent because of the retrospective study design, the limited number of MAC-positive patients with lung cancer detected using bronchoscopy, and the limited patient data available in the medical records. In addition, there was a large population imbalance for the comparisons between MACpositive/other NTM-negative $(n=25)$ and MAC-negative/other NTM-negative $(n=1,223)$ lung cancer patients. Because of this bias, the present data may not be sufficient to definitively elucidate MAC-positivity in patients with lung cancer. Larger epidemiological cohort studies and detailed clinicopathological studies may be required to clarify the relationship between lung cancer and MACLD/MAC infection.

\section{CONCLUSION}

The present study demonstrated an association between MAC infection and the presence of lung cancer. Female sex and advanced age were associated with MAC infection, corroborating previous studies. Lung cancer characteristics, including histological type, location, grade, and clinical stage, were not found to be associated with MAC infection.

$\begin{array}{lll}\text { ABBREVIATIONS } & & \\ \text { CI } & = & \text { Confidence interval } \\ \text { CT } & = & \text { Computed tomography } \\ \text { ILD } & = & \text { Interstitial lung disease } \\ \text { IQR } & = & \text { Interquartile range } \\ \text { MAC } & = & \text { Mycobacterium avium complex } \\ \text { MACLD } & = & \text { MAC lung disease } \\ \text { NTM } & = & \text { Non-tuberculous mycobacteria } \\ \text { NTMLD } & = & \text { NTM-associated lung disease } \\ \text { OR } & = & \text { Odds ratio } \\ \text { PCR } & = & \text { Polymerase chain reaction } \\ \text { TRC } & = & \text { Transcription concerted reaction }\end{array}$

\section{CONFLICT OF INTEREST}

The authors confirm that this article content has no conflict of interest. 


\section{ACKNOWLEDGEMENTS}

Declared none.

\section{REFERENCES}

[1] Morimoto K, Iwai K, Uchimura K, et al. A steady increase in nontuberculous mycobacteriosis mortality and estimated prevalence in Japan. Ann Am Thorac Soc 2014; 11(1): 1-8. [http://dx.doi.org/10.1513/AnnalsATS.201303-067OC] [PMID: 24102151]

[2] Prevots DR, Marras TK. Epidemiology of human pulmonary infection with nontuberculous mycobacteria: a review. Clin Chest Med 2015; 36(1): 13-34. [http://dx.doi.org/10.1016/j.ccm.2014.10.002] [PMID: 25676516]

[3] Prevots DR, Shaw PA, Strickland D, et al. Nontuberculous mycobacterial lung disease prevalence at four integrated health care delivery systems. Am J Respir Crit Care Med 2010; 182(7): 970-6. [http://dx.doi.org/10.1164/rccm.201002-0310OC] [PMID: 20538958]

[4] Simons S, van Ingen J, Hsueh PR, et al. Nontuberculous mycobacteria in respiratory tract infections, eastern Asia. Emerg Infect Dis 2011; 17(3): $343-9$ [http://dx.doi.org/10.3201/eid170310060] [PMID: 21392422]

[5] Sakatani M. The non-tuberculous mycobacteriosis. Kekkaku 2005; 80(1): 25-30. [PMID: 15839060]

[6] Sexton P, Harrison AC. Susceptibility to nontuberculous mycobacterial lung disease. Eur Respir J 2008; $31(6)$ : 1322-33. [http://dx.doi.org/10.1183/09031936.00140007] [PMID: 18515557]

[7] Winthrop KL, McNelley E, Kendall B, et al. Pulmonary nontuberculous mycobacterial disease prevalence and clinical features: an emerging public health disease. Am J Respir Crit Care Med 2010; 182(7): 977-82. [http://dx.doi.org/10.1164/rccm.201003-0503OC] [PMID: 20508209]

[8] Hayashi M, Takayanagi N, Kanauchi T, Miyahara Y, Yanagisawa T, Sugita Y. Prognostic factors of 634 HIV-negative patients with Mycobacterium avium complex lung disease. Am J Respir Crit Care Med 2012; 185(5): 575-83. [http://dx.doi.org/10.1164/rccm.201107-1203OC] [PMID: 22199005]

[9] Tamura A, Hebisawa A, Sagara Y, et al. Pulmonary nontuberculous mycobacteriosis in patients with lung cancer. Kekkaku 2004; 79(6): 367-73.

[PMID: 15293749]

[10] Lande L, Peterson DD, Gogoi R, et al. Association between pulmonary mycobacterium avium complex infection and lung cancer. J Thorac Oncol 2012; 7(9): 1345-51. [http://dx.doi.org/10.1097/JTO.0b013e31825abd49] [PMID: 22699888]

[11] Tamura A, Shimada M, Matsui Y, et al. The value of fiberoptic bronchoscopy in culture-positive pulmonary tuberculosis patients whose prebronchoscopic sputum specimens were negative both for smear and PCR analyses. Intern Med 2010; 49(2): $95-102$. [http://dx.doi.org/10.2169/internalmedicine.49.2686] [PMID: 20075571]

[12] Tanaka E, Amitani R, Niimi A, Suzuki K, Murayama T, Kuze F. Yield of computed tomography and bronchoscopy for the diagnosis of Mycobacterium avium complex pulmonary disease. Am J Respir Crit Care Med 1997; 155(6): 2041-6. [http://dx.doi.org/10.1164/ajrccm.155.6.9196113] [PMID: 9196113]

[13] Sugihara E, Hirota N, Niizeki T, et al. Usefulness of bronchial lavage for the diagnosis of pulmonary disease caused by Mycobacterium avium-intracellulare complex (MAC) infection. J Infect Chemother 2003; 9(4): 328-32. [http://dx.doi.org/10.1007/s10156-003-0267-1] [PMID: 14691654]

[14] Statistics of tuberculosis 2012. $1^{\text {st }}$ ed. Tokyo: Japan Anti-tuberculosis Association 2012; p. 3.

[15] Middleton AM, Chadwick MV, Gaya H. Disinfection of bronchoscopes, contaminated in vitro with Mycobacterium tuberculosis, Mycobacterium avium-intracellulare and Mycobacterium chelonae in sputum, using stabilized, buffered peracetic acid solution ('Nu-Cidex'). J Hosp Infect 1997; 37(2): 137-43. [http://dx.doi.org/10.1016/S0195-6701(97)90183-3] [PMID: 9364262]

[16] Griffith DE, Aksamit T, Brown-Elliott BA, et al. An official ATS/IDSA statement: diagnosis, treatment, and prevention of nontuberculous mycobacterial diseases. Am J Respir Crit Care Med 2007; 175(4): 367-416. [http://dx.doi.org/10.1164/rccm.200604-571ST] [PMID: 17277290]

[17] Han XY, Tarrand JJ, Infante R, Jacobson KL, Truong M. Clinical significance and epidemiologic analyses of Mycobacterium avium and Mycobacterium intracellulare among patients without AIDS. J Clin Microbiol 2005; 43(9): 4407-12. [http://dx.doi.org/10.1128/JCM.43.9.4407-4412.2005] [PMID: 16145084]

[18] Hosoda C, Hagiwara E, Shinohara T, et al. Clinical characteristics of pulmonary Mycobacterium avium complex infection complicated with lung cancer. Kekkaku 2014; 89(8): 691-5. [PMID: 25283009]

[19] Daley CL, Iseman M. Mycobacterium avium complex and lung cancer: chicken or egg? Both? J Thorac Oncol 2012; 7(9): 1329-30 
[http://dx.doi.org/10.1097/JTO.0b013e318265a7ef] [PMID: 22895136]

[20] Middleton AM, Chadwick MV, Nicholson AG, et al. The role of Mycobacterium avium complex fibronectin attachment protein in adherence to the human respiratory mucosa. Mol Microbiol 2000; 38(2): 381-91. [http://dx.doi.org/10.1046/j.1365-2958.2000.02137.x] [PMID: 11069663]

[21] Fujita J, Ohtsuki Y, Shigeto E, et al. Pathological findings of bronchiectases caused by Mycobacterium avium intracellulare complex. Respir Med 2003; 97(8): 933-8. [http://dx.doi.org/10.1016/S0954-6111(03)00120-3] [PMID: 12924521]

[22] West JB, Dollery CT. Distribution of blood flow and ventilation-perfusion ratio in the lung, measured with radioactive carbon dioxide. J Appl Physiol 1960; 15: 405-10. [PMID: 13844133]

[23] Watanabe M, Ishizaka A, Ikeda E, Ohashi A, Kobayashi K. Contributions of bronchoscopic microsampling in the supplemental diagnosis of small peripheral lung carcinoma. Ann Thorac Surg 2003; 76(5): 1668-72. [http://dx.doi.org/10.1016/S0003-4975(03)01015-4] [PMID: 14602308]

[24] Brenner DR, Boffetta P, Duell EJ, et al. Previous lung diseases and lung cancer risk: a pooled analysis from the International Lung Cancer Consortium. Am J Epidemiol 2012; 176(7): 573-85. [http://dx.doi.org/10.1093/aje/kws151] [PMID: 22986146]

[25] Friedrich G. Periphere Lungenkrebse auf dem Borden pleu-ranaher Narben. Virchows Arch 1939; 304: 230-47. [http://dx.doi.org/10.1007/BF02595199]

[26] Bobba RK, Holly JS, Loy T, Perry MC. Scar carcinoma of the lung: a historical perspective. Clin Lung Cancer 2011; 12(3): 148-54. [http://dx.doi.org/10.1016/j.cllc.2011.03.011] [PMID: 21663856]

[27] Tamura A, Hebisawa A, Sagara Y, et al. Coexistence of lung cancer and active pulmonary mycobacteriosis. Kekkaku 2005; 80(5): 413-9. [PMID: 16083049]

[28] Nishimura T, Hasegawa N, Watanabe M, Takebayashi T, Tasaka S, Ishizaka A. Bronchoscopic microsampling to analyze the epithelial lining fluid of patients with pulmonary Mycobacterium avium complex disease. Respiration 2008; 76(3): 338-43. [PMID: 18824886]

[29] Honda JR, Knight V, Watanabe ED. Pathogenesis and risk factors for nontuberculous mycobacterial lung disease. Clin Chest Med 2015; 36: $1-11$. [PMID: 18824886]

(C) Tamura et al.; Licensee Bentham Open.

This is an open access article licensed under the terms of the Creative Commons Attribution-Non-Commercial 4.0 International Public License (CC BY-NC 4.0) (https://creativecommons.org/licenses/by-nc/4.0/legalcode), which permits unrestricted, non-commercial use, distribution and reproduction in any medium, provided the work is properly cited. 